Gazi University
Journal of Science
http://dergipark.gov.tr/gujs

\title{
Characterizations of Bertrand Curves in Finsler 3-Manifold
}

\author{
Fatma ATES ${ }^{1, *}$ (D) Zehra OZDEMIR $^{2}$ (D) F. Nejat EKMEKCI ${ }^{3}$ \\ ${ }^{I}$ Necmettin Erbakan University, Faculty of Science, Department of Mathematics-Computer, 42090, Konya, Turkey \\ ${ }^{2}$ Amasya University, Faculty of Science, Department of Mathematics, 05100, Amasya, Turkey \\ ${ }^{3}$ Ankara University, Faculty of Science, Department of Mathematics, 06100, Ankara, Turkey
}

\section{Highlights}

- We determine the characterization of the Bertrand curve in Finslerian 3-space.

-We produced new Bertrand curves from Finslerian spherical curves.

- We exemplified our results using the Randers metric.

\section{Article Info \\ Received: 20/08/2019 Accepted: 03/04/2020 \\ Keywords \\ Finsler space \\ Indicatrix surface \\ Bertrand curves \\ Randers metric \\ Finsler spherical curve}

\begin{abstract}
In this study, we give the linear condition for the Bertrand curve of a given curve in Finslerian 3space. Using the Izumiya and Takeuchi's paper as an inspiration, Bertrand curves of Finslerian spherical curves are defined according to Randers metric which is a special Finslerian metric. Also, we obtain the Finsler helix derived from the Finslerian circle according to Randers metric and their visualizations are given by using the Mathematica program.
\end{abstract}

\section{INTRODUCTION}

In 1917, Paul Finsler introduced the curves and surfaces by using the minkowski norm instead of inner product in doctoral thesis after him called Finsler geometry. The author defines Finsler geometry as the natural generalization of the Riemannian geometry. Therefore, this geometry includes the Riemannian metric. Finsler geometry is an important system which use in thermodynamics, optics, ecology, evolution, biology, etc. For example, the Finsler distance on this structure corresponds to the phase of an optical waveguide. Also, Finsler geometry appears in field theory and particle physics. Besides, it has an extraordinary research field in geometry, biology, physics, engineering, and computer sciences [1-5]. Ergüt et al. studied $A W(k)$ - type curves in three dimensional Finsler manifold in [6]. Remizov [7] investigated the singularities of geodesics flows in two-dimensional Finsler space. Yıldırım et al. defined the helices in Finsler space and give some characterizations of these curves in $[8,9]$.

G. Randers in 1941 first introduced the Randers metric that is outlined as a special Finsler metric in [10]. Randers metric is composed of two parts: an Euclidean norm on a vector space $R^{n}$ and $\beta(x)=b_{i}(x) y^{i}$ be a linear form on $R^{n}$. Randers spaces must be required to have Riemannian norm strictly smaller than 1 everywhere, i.e., $\|\beta\|_{\gamma}:=\sqrt{a^{i j} b_{i} b_{j}}<1$.

Bertrand curve is first expressed by J. Bertrand in 1850 in [11]. A curve is called as a Bertrand mate of the other curve if its principal normal vector is normal to other curve. The curvature and torsion of the Bertrand 
curve is satisfied the linear combination $a \kappa+b \tau=1$ such $a, b$ constants. Then, Izumiya and Takeuchi [12] found a new method for the generation of Bertrand curve. They obtained a new Bertrand curve from a given spherical curve in Euclidean 3-space. Babaarslan [13] showed that Bertrand curves can be constructed from unit speed curves on Euclidean 2-sphere $S^{2}$. Also, he gave a relation between Bertrand curves and helices.

In this paper, we examine the characterization of the Bertrand curves in Finsler space. Then, we find the Bertrand curves derived from curves on the indicatrix surface which is sphere of Finsler space. Also, we visualized an example using the Randers metric which is a special Finslerian metric.

\section{PRELIMINARIES}

In this section, we give the main geometric objects necessary for the study of Finsler geometry.

Definition 1. Let $M^{\prime}$ be non-empty open submanifold of $T M$ such that $\pi\left(M^{\prime}\right)=M$ and $\theta(M) \cap M^{\prime}=\varphi$, where $\theta$ is a zero section of $T M$. It is given a smooth function $F: T M \rightarrow(0, \infty)$. Then suppose that any coordinate system $\left\{\left(U^{\prime}, \Phi^{\prime}\right) ; x^{i}, y^{i}\right\}$ in $M^{\prime}$, the following conditions are fulfilled:

$\left(\boldsymbol{F}_{1}\right) F$ is positively homogeneous of degree one, that is, $F(\lambda X)=\lambda F(X)$ for all positive $\lambda>0$, and $X \in T M$ or in the coordinate system, we have

$$
F\left(x^{1}, \ldots x^{m}, \lambda y^{1}, \ldots, \lambda y^{m}\right)=\lambda F\left(x^{1}, \ldots x^{m}, y^{1}, \ldots, y^{m}\right),
$$

for any $(x, y) \in \Phi^{\prime}\left(U^{\prime}\right)$ and any $\lambda>0$.

$\left(\boldsymbol{F}_{2}\right)$ For any nonzero $V \in T_{x} M$, the following quadratic form $g_{V}$ on $T_{x} M$

$$
\begin{gathered}
g_{V}: T_{x} M \times T_{x} M \rightarrow \mathrm{R}, \\
g_{V}(X, Y)=\langle X, Y\rangle_{V}:=\frac{1}{2} \frac{\partial^{2}}{\partial s \partial t}\left[F^{2}(V+s X+t Y)\right]_{\mid s=t=0}
\end{gathered}
$$

is a positive definite quadratic form on $\mathrm{R}^{m}$ and using the canonical coordinates

$(x, y)=\left(x^{1}, \ldots, x^{n}, y^{1}, \ldots, y^{n}\right)$ on $T M$, the coefficients of the metric $g$ can be stated as follows

$$
g_{i j}(x, y)=\frac{1}{2} \frac{\partial^{2} F^{2}}{\partial y^{i} \partial y^{j}}(x, y), i, j \in\{1, \ldots, m\}
$$

The triple $\mathrm{F}^{m}=\left(M, M^{\prime}, F\right)$ with $F$ satisfying $\left(\boldsymbol{F}_{\mathbf{1}}\right)$ and $\left(\boldsymbol{F}_{\mathbf{2}}\right)$ is a Finsler manifold and $F$ is called the fundamental function of $\mathrm{F}^{m}$.

Let $\mathrm{F}^{m+1}=\left(M, M^{\prime}, F\right)$ be a Finsler manifold and $\mathrm{F}^{1}=\left(c, c^{\prime}, F_{1}\right)$ be a one dimensional Finsler submanifold of $\mathrm{F}^{m+1}$. Here, $c$ is a smooth curve in $M$ and $c^{\prime}$ is the tangent bundle of the curve $c$ in $M^{\prime}$. The curve $c$ has the following representation

$$
x^{i}=x^{i}(s): \quad i \in\{1,2, \ldots, m+1\}, \quad s \in(a, b)
$$


where $(s, v)$ denoted by the coordinates on $c^{\prime}$ and $s$ is the arc length parameter of the curve $c$. Let $(s, v)$ be local coordinates and $\left\{\frac{\partial}{\partial s}, \frac{\partial}{\partial v}\right\}$ be a natural frame on $c^{\prime}$ where $\frac{\partial}{\partial v}$ is a unit Finslerian vector field. The vertical covariant derivative of the tangent vector $\frac{\partial}{\partial v}$ of the curve $c$ that is $g\left(\frac{\partial}{\partial v}, \frac{\partial}{\partial v}\right)=1$. The following equalities are given

$$
\frac{\partial}{\partial s}=\frac{d x^{i}}{d s} \frac{\partial}{\partial x^{i}}+v \frac{d^{2} x^{i}}{d s^{2}} \frac{\partial}{\partial y^{i}} \text { and } \frac{\partial}{\partial v}=\frac{d x^{i}}{d s} \frac{\partial}{\partial y^{i}}
$$

These imply following equation

$$
y^{i}(s, v)=v \frac{d x^{i}}{d s}, i \in\{0, \ldots, m\}
$$

where $\left\{\frac{\partial}{\partial s}, \frac{\partial}{\partial v}\right\}$ is the natural frame on $c^{\prime}$.

A Finsler vector field $X$ on $\mathrm{F}^{m+1}$ along $c^{\prime}$ is projectable on $c$. Then, it can be expressed as follows:

$$
X\left(x(s), v x^{\prime}(s)\right)=X^{i}(s) \frac{\partial}{\partial y^{i}}\left(x(s), v x^{\prime}(s)\right)
$$

at any point $\left(x(s), v x^{\prime}(s)\right) \in c^{\prime}$. From here, a vector field $X^{*}$ on $c$ denoted by the following formula

$$
X^{*}(x(s))=X^{i}(s) \frac{\partial}{\partial x^{i}}(x(s))
$$

Thus, the vector field $X^{*}(x(s))$ considered as the projection of the Finsler vector $X\left(x(s), v x^{\prime}(s)\right)$ on the tangent bundle $T M$ of $M$ at $x(s) \in c$ (see for details [3]). The covariant derivatives according to Cartan connection of any projectable Finsler vector field $X$ in the direction of $\frac{\partial}{\partial v}$ vanish identically on $c^{\prime}$.

$$
\left(\nabla_{\frac{\partial}{\partial v}} X\right)\left(x(s), v x^{\prime}(s)\right)=0, s \in(-\varepsilon, \varepsilon) \text { and }
$$

and

$$
\nabla_{\frac{\partial}{\partial v}} \frac{\partial}{\partial v}=0
$$

enable us to express that the vertical covariant derivatives along $c$ with respect to Cartan connection don't give any Frenet frame for $c$. So, that the non-linear connection $H c^{\prime}$ on $\mathrm{F}^{1}$ by the canonical non-linear connection $G M^{\prime}$ of $\mathrm{F}^{m+1}$ is locally spanned by $\frac{\partial}{\partial s}$. The Cartan connection is the best choice for studying the geometry of curves in a Finsler manifold. The Cartan derivative of the vector field $\frac{\partial}{\partial v}$ is obtained as folows: 


$$
\nabla_{\partial / \partial s}^{*} \frac{\partial}{\partial v}=\left(\frac{d^{2} x^{k}}{d s^{2}}+2 G^{k}(s)\right) \frac{\partial}{\partial y^{k}}
$$

where $G^{i}=\frac{g^{i l}}{4}\left\{\frac{\partial^{2} F^{2}}{\partial x^{m} \partial y^{1}} y^{m}-\frac{\partial F^{2}}{\partial x^{l}}\right\}$ and $g^{i j}=\left(g_{i j}\right)^{-1}$.

Let $c=c(s)$ be a smooth curve in $\mathrm{F}^{3}$ and $s$ be an arc length parameter of the curve $c$. Suppose that the moving Frenet frame along the curve $c$ denoted by $\left\{T:=\frac{\partial}{\partial v}, N, B\right\}$ in the Finslerian 3 -space. Then, the Frenet formulas of the curve $c$ are given by

$$
\begin{gathered}
\nabla_{\frac{\partial}{\partial s}}^{*} T(s)=\kappa(s) N(s), \\
\nabla_{\frac{\partial}{\partial s}}^{*} N(s)=-\kappa(s) T(s)+\tau(s) B(s), \\
\nabla_{\frac{\partial}{\partial s}}^{*} B(s)=-\tau(s) N(s),
\end{gathered}
$$

where the Frenet vectors $N(s)$ and $B(s)$ are Finslerian principal normal and binormal vectors of the curve $c$, respectively. The Finslerian curvature and torsion of the curve $c$ are defined by

$$
\begin{gathered}
\kappa(s)=\left\{g_{i j}(s)\left(c^{\prime \prime}(s)+2 G^{i}(s)\right)\left(c^{\prime \prime}(s)+2 G^{j}(s)\right)\right\}^{\frac{1}{2}} \\
\tau(s)=-g\left(\nabla_{\frac{\partial}{\partial s}}^{*} N, B\right)(s)=-g_{i j}(s) B^{i}(s)\left\{\frac{\partial N^{j}}{\partial s}+N^{k}(s) S_{k}^{j}(s)\right\}
\end{gathered}
$$

[3].

\section{BERTRAND CURVES IN FINSLER 3-MANIFOLD}

Definition 2. [11] Let $c(s)$ and $c^{*}\left(s^{*}\right)$ be unit speed curves in $3-$ dimensional Finsler manifold $\mathrm{F}^{3}=$ $\left(M, M^{\prime}, F\right)$. These curves are called the Bertrand curve pair, if there is a relationship in which the principal normals of these curves are parallel to each other at the opposite points.

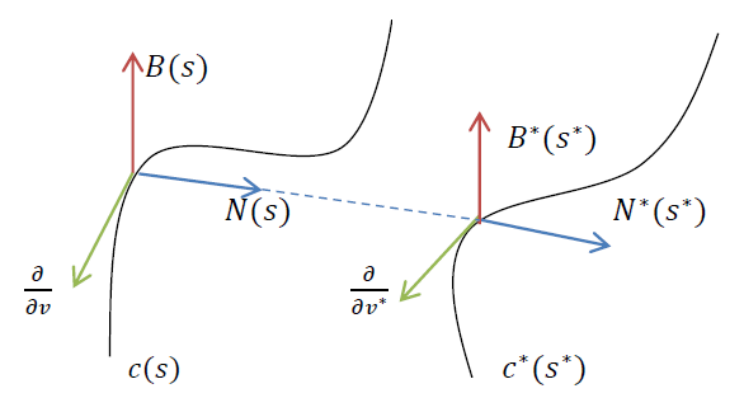

Figure 1. Bertrand curves $c(s)$ and $c^{*}\left(s^{*}\right)$

From the Figure 1, the relationship between the curves $c$ and $c^{*}$ is given by

and using Equation (5), we can calculate

$$
c^{*}\left(s^{*}\right)=c(s)+\lambda(s) N(s) .
$$

$$
\nabla_{\frac{\partial}{\partial s}}^{*} c^{*}\left(s^{*}\right)=(1-\lambda(s) \kappa(s)) T(s)+\left(\nabla_{\frac{\partial}{\partial s}}^{*} \lambda(s)\right) N(s)+\lambda(s) \tau(s) B(s)
$$


and since the tangent vector $\frac{\partial}{\partial v^{*}}$ of $c^{*}$ and the principal normal vector of $c$ are orthogonal then it is calculated that $\lambda$ is a constant. Take into account the constant $\lambda$, the Frenet vectors of the curve $c^{*}$ is obtained in terms of Frenet vectors of the curve $c$ as follows:

$T^{*}\left(s^{*}\right)=\frac{1}{\sqrt{\left((1-\lambda \kappa(s))^{2}\right)+(\lambda(s) \tau(s))^{2}}}((1-\lambda \kappa(s)) T(s)+\lambda \tau(s) B(s))$,

$N^{*}\left(s^{*}\right)=N(s)$

$B^{*}\left(s^{*}\right)=T^{*}\left(s^{*}\right) \times N^{*}\left(s^{*}\right)$,

where $d s^{*}=\sqrt{\left((1-\lambda \kappa(s))^{2}\right)+(\lambda(s) \tau(s))^{2}} d s$.

Definition 3. [14] In Finsler space, $\mathrm{F}^{n}=\left(M, M^{\prime}, F\right)$ angle $\alpha$ usually means the angle of the two vectors $\xi(p, y)$ and $\eta(p, y)$ of $V_{(p, y)} T M$. The cosine of the angle $\alpha$ is defined by

$$
\cos \alpha=\frac{g_{i j}(p, y) \xi^{i}(p, y) \eta(p, y)}{F(\xi) F(\eta)} .
$$

Theorem 1. Let $c:=c(s)$ and $c^{*}:=c^{*}\left(s^{*}\right)$ be curves with non-zero curvatures in the 3 -dimensional Finsler space. Then, $c^{*}$ is the Bertrand mate of the curve $c$ if and only if the curvature and torsion of the curve $c$ provides the linear relation

$$
c_{1} \kappa(s)+c_{2} \tau(s)=1
$$

where $c_{1}=\lambda, c_{2}=\lambda \cot \theta$ are constants and $\theta$ is angle between tangent vector fields of the curves $c$ and $c^{*}$. The curvatures of the curve $c$ are defined by $\kappa(s)=\left\{g_{i j}(s)\left(c^{\prime \prime} i(s)+2 G^{i}(s)\right)\left(c^{\prime \prime} j(s)+2 G^{j}(s)\right)\right\}^{\frac{1}{2}}$ and $\tau(s)=-g_{i j}(s) B^{i}(s)\left\{\frac{\partial N^{j}}{\partial s}+N^{k}(s) S_{k}^{j}(s)\right\}$.

Proof. Let $c$ and $c^{*}$ be Bertrand mate curves in 3 - dimensional Finsler space. If cosine of the angle between their tangent vectors $T(s)$ and $T^{*}\left(s^{*}\right)$ is $\cos \theta=\frac{g\left(T(s), T^{*}\left(s^{*}\right)\right)}{\left.F(T(s)) F\left(T^{*}\left(s^{*}\right)\right)\right)}$, then we can write

$$
T^{*}\left(s^{*}\right)=\cos \theta T(s)+\sin \theta B(s) .
$$

Differentiating Equation (7) with respect to $s$ and using the Frenet formulas in Equation (5), we get

$$
\nabla_{\frac{\partial}{\partial s}}^{*} T^{*}\left(s^{*}\right)=-\left(\nabla_{\frac{\partial}{\partial s}}^{*} \theta\right) \sin \theta T(s)+(\kappa(s) \cos \theta-\tau(s) \sin \theta) N(s)+\left(\nabla_{\frac{\partial}{\partial s}}^{*} \theta\right) \cos \theta B(s) .
$$

Since $g\left(N^{*}\left(s^{*}\right), T(s)\right)=0$, we obtain that the angle $\theta$ is a constant. Since it can be written the tangent vector field of the curve $c^{*}$ in terms of Frenet frame vectors of the curve $c$ as follows:

$$
T^{*}\left(s^{*}\right)=\frac{1}{\sqrt{(1-\lambda \kappa(s))^{2}+(\lambda \tau(s))^{2}}}\{(1-\lambda \kappa(s)) T(s)+\lambda \tau(s) B(s)\} .
$$

Considering the last equation and Equation (7), the following ratio is obtained

$$
\frac{1-\lambda \kappa(s)}{\cos \theta}=\frac{\lambda \tau(s)}{\sin \theta} \text {. }
$$

The last equality gives desired result for some constants $c_{1}=\lambda$ and $c_{2}=\lambda \cot \theta$. 
Conversely, we assume that $c(s)$ is a smooth curves in $\mathrm{F}^{3}$ and the equality $\lambda \kappa(s)+\lambda \cot \theta \tau(s)=1$ is satisfied for some constants $\lambda$ and $\theta$. The curve $c^{*}$ is defined by

$$
c^{*}\left(s^{*}\right)=c(s)+\lambda N(s) \text {. }
$$

From the last equality, the principal normal vector field $N^{*}$ of the curve $c^{*}$ is equal to the principal normal vector field $N$ of the curve $c$, i.e. $N^{*}=N$. So, the curve $c^{*}$ is the Bertrand curve of the curve $c$.

\section{CONSTRUCTION OF BERTRAND CURVES IN FINSLER 3-SPACE}

In this section, we will first define the indicatrix hypersurface in Finsler manifold. Then, we will define the Darboux frame for the curves that lie on the indicatrix surface. We will build the Bertrand curve from the Finsler spherical curves by using Izumiya and Takeuchi's method. Finally, we will construct Bertrand curves using the Finslerian spherical images of a given curve in Finsler space.

Definition 4. [14] In the Finsler manifold $F^{m+1}=\left(M, M^{\prime}, F\right)$, the indicatrix hypersurface which plays the role of the Euclidean unit sphere $S^{n-1} \in E^{n}$ is defined by

$$
I\left(p_{0}\right)=\left\{y \in T_{p_{0}} M \mid F\left(p_{0}, y\right)=1\right\},
$$

$I$ is strictly convex neighborhood of point $p_{0}$, that is $F\left(p_{0}, y\right)=F\left(p_{0},-y\right)$ for all $\left(p_{0}, y\right)$.

Theorem 2. Let $c$ be a smooth curve with arc length parameter $s$ on the indicatrix surface $F S^{2}:=I(0)$ in the Finsler space $F^{3}$. The curve $c$ has the Darboux frame $\left\{c(s), T(s)=\frac{\partial}{\partial v}, Y(s)=c(s) \times T(s)\right\}$ and geodesic curvature function $\kappa_{g}(s)=g\left(\nabla^{*} \frac{\partial}{\partial s} T(s), Y(s)\right)$. The derivatives of these vectors in terms of themselves is to be formulated as follows:

$$
\begin{gathered}
\nabla_{\frac{\partial}{\partial s}}^{*} c(s)=T(s), \\
\nabla_{\frac{\partial}{\partial s}}^{*} T(s)=-c(s)+\kappa_{g}(s) Y(s), \\
\nabla_{\frac{\partial}{\partial s}}^{*} Y(s)=-\kappa_{g}(s) T(s),
\end{gathered}
$$

where $\kappa_{g}(s)=\left\{g_{i j}(s)\left(c^{\prime \prime \prime}(s)+2 G^{i}(s)\right)\left(c^{\prime \prime j}(s)+2 G^{j}(s)\right)-1\right\}^{\frac{1}{2}}$ is geodesic curvature of the spherical curve $c(s)$.

Proof. Let $c: I \rightarrow F S^{2}:=I(0)$ be a Finslerian spherical curve with arc length parameter $s$. Assume that the curve $c$ has orthonormal triple vectors $\left\{c(s), T(s)=\frac{\partial}{\partial v}, Y(s)=c(s) \times T(s)\right\}$ on the indicatrix surface $F S^{2}$ at the point zero. Then we can calculate the derivations of these vectors as follows: Since the curve $c$ is a unit speed curve then, we get $\nabla_{\frac{\partial}{\partial s}}^{*} c(s)=T(s)$. Also, the derivation of the tangent vector $T(s)$ is written in terms of the triple vector fields as $\nabla_{\frac{\partial}{\partial s}}^{*} T(s)=x_{1}(s) c(s)+x_{2}(s) T(s)+x_{3}(s) Y(s)$. Since $g(c(s), c(s))=1$ we obtain $g\left(\nabla_{\frac{\partial}{\partial s}}^{*} T(s), c(s)\right)=-1$, that is $x_{1}(s)=-1 . x_{3}(s)=g\left(\nabla_{\frac{\partial}{\partial s}}^{*} T(s), Y(s)\right)$ can be defined by the geodesic curvature $\kappa_{g}(s)$. So, it is found the second equality in the Equation (9). The vector field $Y(s)=\left(y^{1}(s), y^{2}(s), y^{3}(s)\right)$ is introduced by the following components

$$
y^{1}(s)=\left|\begin{array}{cc}
c_{2}(s) & c_{3}(s) \\
c^{\prime}{ }_{2}(s) & c_{3}{ }^{\prime}(s)
\end{array}\right|, y^{2}(s)=\left|\begin{array}{cc}
c_{3}(s) & c_{1}(s) \\
c^{\prime}{ }_{3}(s) & c_{1}^{\prime}(s)
\end{array}\right|, y^{3}(s)=\left|\begin{array}{cc}
c_{1}(s) & c_{2}(s) \\
c^{\prime}{ }_{1}(s) & c_{2}{ }^{\prime}(s)
\end{array}\right|
$$

and $Y(s)=g_{i j} y^{i}(s) y^{j}(s)$. If we consider the expression of the vector $Y(s)$ in the equation $\kappa_{g}(s)=$ $g\left(\nabla_{\frac{\partial}{\partial s}}^{*} T(s), Y(s)\right)$, then we obtain the geodesic curvature of the Finslerian spherical curve $c$ as follows 


$$
\kappa_{g}(s)=\left\{g_{i j}(s)\left(c^{\prime \prime i}(s)+2 G^{i}(s)\right)\left(c^{\prime \prime j}(s)+2 G^{j}(s)\right)-1\right\}^{\frac{1}{2}} .
$$

If we take $\nabla_{\frac{\partial}{\partial s}}^{*} Y(s)=a_{1}(s) c(s)+a_{2}(s) T(s)+a_{3}(s) Y(s)$, then we calculate $a_{1}(s)=0, a_{2}(s)=$ $-\kappa_{g}(s)$ and $a_{3}(s)=0$ by using the similar method as above .

\subsection{Bertrand Curve of a Given Curve on Indicatrix Surface According to Randers Metric}

In this subsection, the theorems and examples are given according to the Randers metric which is special Finslerian metric defined by Randers. The Randers metric defines as

$$
F(x, y):=\alpha(x, y)+\beta(x, y) .
$$

Here, $\alpha(x, y)=\sqrt{a_{i j}(x) y^{i} y^{j}}$ is Euclidean norm. The authors [15] gave the Randers metric as follows:

$$
g_{i j}=\frac{F}{\alpha}\left\{a_{i j}-\frac{y^{i}}{\alpha} \frac{y^{j}}{\alpha}+\frac{\alpha}{F}\left(b_{i}+\frac{y_{i}}{\alpha}\right)\left(b_{j}+\frac{y_{j}}{\alpha}\right)\right\}
$$

where $y_{i}:=a_{i j} y^{j}$. Since the bilinear form $\left(g_{i j}\right)$ is positive definite, then the length of $\beta$ is less then 1 , i.e., $\|\beta\|_{\gamma}:=\sqrt{a^{i j} b_{i} b_{j}}<1$ where $\left(a^{i j}\right):=\left(a_{i j}\right)^{-1}$.

Theorem 3. Let $c: I \rightarrow F S^{2}$ be a Finslerian spherical curve with arc length parameter $s$. Bertrand mate of the curve $c$ is given by

$$
\tilde{c}(t)=a \int_{0}^{t} c(u) d u+\operatorname{atan} \xi \int_{0}^{t} Y(u) d u
$$

where $a$ and $\xi$ are constant real numbers.

Proof. Using the Frenet formulas in the Equation (5), we can calculate

$$
\begin{gathered}
\nabla_{\frac{\partial}{\partial t}}^{*} \tilde{c}=a(c(t)+\tan \xi Y(t)), \\
\nabla_{\frac{\partial}{\partial t}}^{*} \nabla_{\frac{\partial}{\partial t}}^{*} \tilde{c}=a\left(1-\tan \xi \kappa_{g}(t)\right) T(t),
\end{gathered}
$$

$\nabla_{\frac{\partial}{\partial t}}^{*} \frac{\partial}{\partial t} \nabla_{\frac{\partial}{\partial t}}^{*} \tilde{c}=-a\left(1-\tan \xi \kappa_{g}(t)\right) c(t)+a\left(-\tan \xi \nabla_{\frac{\partial}{\partial t}}^{*} \kappa_{g}(t)\right) T(t)+a\left(1-\tan \xi \kappa_{g}(t)\right) \kappa_{g}(t) Y(t)$

From the Equation (11), we can calculate the following equality

$$
\nabla_{\frac{\partial}{\partial t}}^{*} \tilde{c} \times \nabla_{\frac{\partial}{\partial t}}^{*} \nabla_{\frac{\partial}{\partial t}}^{*} \tilde{c}=a^{2}\left(1-\tan \xi \kappa_{g}(t)\right)(-\tan \xi c(t)+Y(t))
$$

and the Randers norm of this vector is calculated as follows:

$$
F\left(\nabla_{\frac{\partial}{\partial t}}^{*} \tilde{c} \times \nabla_{\frac{\partial}{\partial t}}^{*} \nabla_{\frac{\partial}{\partial t}}^{*} \tilde{c}\right)=a^{2}\left(1-\tan \xi \kappa_{g}(t)\right) \sec \xi .
$$

Take into account the Equations (11)-(13) in the curvature formulas

$$
\tilde{\kappa}(s)=\frac{F\left(\nabla_{\frac{\partial}{\partial t}}^{*} \tilde{c} \times \nabla_{\frac{\partial}{\partial t}}^{*} \nabla_{\frac{\partial}{\partial t}}^{*} \tilde{c}\right)}{F^{3}\left(\nabla_{\frac{\partial}{\partial t}}^{*} \tilde{c}\right)} \text { and } \tilde{\tau}(s)=\frac{g\left(\nabla_{\frac{\partial}{\partial t}}^{*} \tilde{c} \times \nabla_{\frac{\partial}{\partial t}}^{*} \nabla_{\frac{\partial}{\partial t}}^{*} \tilde{c}, \nabla_{\frac{\partial}{\partial t}}^{*} \nabla_{\frac{\partial}{\partial t}}^{*} \nabla_{\frac{\partial}{\partial t}}^{*} \tilde{c}\right)}{F^{2}\left(\nabla_{\frac{\partial}{\partial t}}^{*} \tilde{c} \times \nabla_{\frac{\partial}{\partial}}^{*} \nabla_{\frac{\partial}{\partial t}}^{*} \tilde{c}\right)}
$$

we obtain the curvature and the torsion of the curve $\tilde{c}$ :

$$
\tilde{\kappa}(s)=\frac{\cos ^{2} \xi}{a}\left(1-\tan \xi \kappa_{g}(s)\right) \text { and } \tilde{\tau}(s)=\frac{\cos ^{2} \xi}{a}\left(\tan \xi+\kappa_{g}(s)\right),
$$

respectively. Since the curvatures of the curve $\tilde{c}$ satisfy the Equation (14), we can say the curve $\tilde{c}$ is Bertrand mate of the Finslerian spherical curve $c$. 
Corollary 1. Let $c: I \rightarrow F S^{2}$ be a smooth curve with arc length parameter $s$. The curve $c$ is a circle if and only if its Bertrand curve $\tilde{c}$ is an circular helix.

Proof. The curve $\tilde{c}$ is a circle its geodesic curvature $\kappa_{g}(s)$ is a constant, that is, $\kappa_{g}{ }^{\prime}(s)=0$. Considering the last equality with the Equation (14), we obtain the curvatures $\tilde{\kappa}$ and $\tilde{\tau}$ are constants. This is completed the proof.

Example. Let $c$ be an ellipse on the Finslerian $2-$ sphere $F S^{2}$ according to Randers norm $F\left(u_{1}, u_{2}, u_{3}\right)=\sqrt{\left(u_{1}\right)^{2}+\left(u_{2}\right)^{2}+\left(u_{3}\right)^{2}}+b . u_{1}$. The curve $c$ on $F S^{2}$ is parameterized as

$$
c(s)=\left(\frac{\cos s-b}{1-b^{2}}, \frac{\sin s}{\sqrt{1-b^{2}}}, 0\right)
$$

where $b \in(0,1)$. The Bertrand curve $\tilde{c}$ in Figure 2 (right) related to the curve $c(s)$ obtained as the form

$$
\tilde{c}(s)=\left(\frac{\sin s-b s}{1-b^{2}},-\frac{\cos s}{\sqrt{1-b^{2}}}, s+c_{1}\right) \text {. }
$$
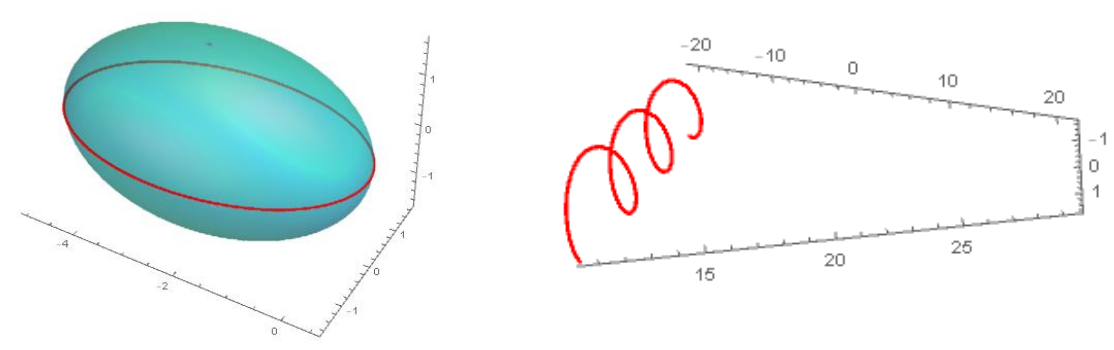

Figure 2. Ellipse curve and Bertrand curve derived from it

\subsection{Bertrand Curves of Finslerian Spherical Image Curves According to Randers Metric}

Definition 5. The locus of points whose position vector is the vector $T(s)$ along to the curve $c$ is called the tangent Finslerian spherical image curve $c_{T}$ of the curve $c$.

Theorem 4. Let $c_{T}: I_{T} \subset I \rightarrow F S^{2}$ be the tangent Finslerian spherical curve of the curve $c$. The Bertrand mate of the curve $c_{T}$ is given by

$$
\tilde{c}_{T}(t)=\operatorname{ac}(t)+\operatorname{atan} \xi \int_{0}^{s_{T}} B(u) d u
$$

where $s_{T}=\int \kappa(s) d s$ and $a, \xi$ are constants.

Proof. Let $c: I \rightarrow F^{3}, c:=c(s)$ be a smooth curve with Frenet frame apparatus $\{T(s), N(s), B(s), \kappa(s), \tau(s)\}$ in 3 -dimensional Finsler space and $s$ be arc length parameter of the curve $c$. Let $c_{T}\left(s_{T}\right)$ be the tangent Finslerian spherical image of the curve $c$ where $\varphi: I \rightarrow I_{T}, s_{T}=\varphi(s)$ is a regular $C^{\infty}$-function. The Darboux frame of the curve $c_{T}$ is defined by $\left\{c_{T}=T(s), T_{T}=N(s), Y_{T}=\right.$ $B(s)\}$. If these vectors are considered in the Equation (10), then it is obtained Bertrand mate of the tangent Finslerian spherical image curve $c_{T}$ as in Equation (15).

Definition 6. The locus of points whose position vector is the vector $N(s)$ along to the curve $c$ is called the normal Finslerian spherical image curve $c_{N}$ of the curve $c$.

Theorem 5. Let $c_{N}: I_{N} \subset I \rightarrow F S^{2}$ be the normal Finslerian spherical curve of the curve $c$ with the Darboux frame $\left\{c_{N}, T_{N}=N, Y_{N}=c_{N} \times T_{N}\right\}$. The Bertrand curve of the curve $c_{N}$ is given by 


$$
\tilde{c}_{N}(t)=a \int_{0}^{t} c_{N}(u) d u+\operatorname{atan} \xi \int_{0}^{s_{N}} Y_{N}(u) d u
$$

where $s_{N}=\int \sqrt{\kappa^{2}(s)+\tau^{2}(s)} d s$ and $a, \xi$ are constants.

Proof. Let $c_{N}\left(s_{N}\right)$ be the normal Finslerian spherical image of the curve $c$ where $\varphi: I \rightarrow I_{N}, s_{N}=\varphi(s)$ is a regular $C^{\infty}$-function. The Darboux frame vectors of the curve $c_{N}$ in terms of the Frenet vectors of the curve $c$ are

$$
\begin{aligned}
& c_{N}=N(s), \\
& T_{N}=\frac{1}{\sqrt{\kappa^{2}(s)+\tau^{2}(s)}}(-\kappa(s) T(s)+\tau(s) B(s)), \\
& Y_{N}=\frac{1}{\sqrt{\kappa^{2}(s)+\tau^{2}(s)}}(\tau(s) T(s)+\kappa(s) B(s)) .
\end{aligned}
$$

If these vectors are considered in the Equation (10), then it is obtained Bertrand mate of the normal Finslerian spherical image curve $c_{N}$ as in Equation (16).

Definition 7. The locus of points whose position vector is the vector $B(s)$ along to the curve $c$ is called the binormal Finslerian spherical image $c_{B}$ of the curve $c$.

Theorem 6. Let $c_{B}: I_{B} \subset I \rightarrow F S^{2}$ be the normal Finslerian spherical curve of the curve $c$ with the Darboux frame $\left\{c_{B}=B(s), T_{B}, Y_{B}=c_{B} \times T_{B}\right\}$. The Bertrand curve corresponding to the binormal Finslerian spherical curve is given by

$$
\tilde{c}_{B}(t)=a \int_{0}^{t} B(u) d u+\operatorname{atan} \xi c\left(s_{B}\right)
$$

where $s_{B}=\int \tau(s) d s$ and $a, \xi$ are constants.

Proof. In [16], the tangent vector of the binormal Finslerian spherical image curve was calculated by $T_{B}=-N(s)$. So, the Darboux frame vectors of the curve $c_{B}$ are obtained by $c_{B}=B(s), T_{B}=-N(s)$ and $Y_{B}=T(s)$. If these vectors are considered in the Equation (10), then it is obtained Bertrand curve of the binormal Finslerian spherical curve $c_{B}$ as in Equation (17).

\section{CONCLUSION}

In this study, the construction of the Bertrand curve from a given curve is discussed in Finslerian 3 -space. Bertrand pair of spherical curves according to the Randers metric which is a special Finsler metric is characterized in the Equation (10). Then, the Bertrand pair of the Finslerian spherical image curves is obtained by calculating the Darboux frames of these special curves according to the Randers metric. The Finsler helix is generated using the ellipse curve which is the circle of the Randers metric and their visualization is shown with the help of the Mathematica program.

\section{ACKNOWLEDGEMENT}

The authors would like to thank referee(s) for their valuable suggestions and comments that helped to improve the presentation of this paper. This paper was presented in the 14th Ankara Mathematics Days Symposium held on June 28-29, 2019.

\section{CONFLICTS OF INTEREST}

No conflict of interest was declared by the authors.

\section{REFERENCES}

[1] Akbar-Zadeh, H., "Initiation to global Finslerian geometry”, North-Holland Math Library, (2006). 
[2] Asanjarani, A., Bidabad, B., "Classification of complete Finsler manifolds through a second order differential equation", Differential Geometry and its Applications, 26: 434-444, (2008).

[3] Bejancu, A., Farran, H.R., "Geometry of pseudo-Finsler submanifolds”, Kluwer Academic Publishers, (2000).

[4] Bidabad, B., Shen, Z., "Circle-preserving transformations in Finsler spaces", Publicationes Mathematicae Debrecen, 81: 435-445, (2012).

[5] Shen, Z., "Lecture on Finsler geometry", World Scientific Publishing Company, (2001).

[6] Ergut, M., Kulahci M., "Special curves in three dimensional Finsler manifold $F^{3}$ ", TWMS Journal of Pure and Applied Mathematics, (5)2: 147-151, (2014).

[7] Remizov, A.O., "Geodesics in generalized Finsler spaces: singularities in dimension two", Journal of Singularities, 14: 172-193, (2016).

[8] Yildirim, M.Y., Bektas, M., "Helices of the 3-dimensional Finsler manifold", Journal of Advanced Mathematical Studies, 2(1): 107-113, (2009).

[9] Yildirim, M.Y., "Biharmonic general helices in 3 -dimensional Finsler manifold", Karaelmas Science and Engineering Journal, 7(1): 1-4, (2017).

[10] Randers, G., "On an asymmetric metric in the four-space of general relativity", Physical Review Journals, 59: 195-199, (1941).

[11] Bertrand, J.M., "Memoire sur la theorie des courbes a double courbure", Journal de Mathematiques Pures et Appliquees, 15: 332-350, (1850).

[12] Izumiya, S., Takeuchi, N., "Generic properties of helices and Bertrand curves", Journal of Geometry, 74: 97-109, (2002).

[13] Babaarslan, M., "Constant slope surfaces and their applications", P.hD. thesis, Ankara University, (2013).

[14] Tamassy, L., "Direct geometrical method in Finsler geometry", Variations, Geometry and Physics, (17): 293-314, (2009).

[15] Chern, S.S., Shen, Z., “Riemann Finsler Geometry”, World Scientific, (2005).

[16] Ates, F., Ozdemir, Z., Ekmekci, N., "Special curves in Finsler space", Proceedings of the Institute of Mathematics and Mechanics, 44(2): 198-208, (2018). 